\title{
September/October 2012
}

SurfNet-Technology of the Future-Today!

Vol 3, Issue 5

\section{C4: A Creative-Coding API for Media, Interaction, and Animation}

\section{By Travis Kirton, Sebastian Boring, Dominikus Baur, Lindsay MacDonald, and Sheelagh Carpendale}

Although there has been widespread proliferation of creativity-support programming languages, the design of many toolkits and application programming interfaces (APIs) for expression and interactivity do not take full advantages of the unique space of mobile multitouch devices. In designing a new API for this space we first consider five major problem spaces and present an architecture that attempts to address these to move beyond the low-level manipulation of graphics giving first-class status to media objects.

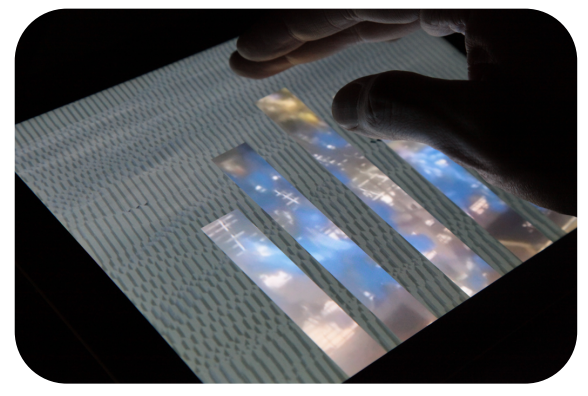

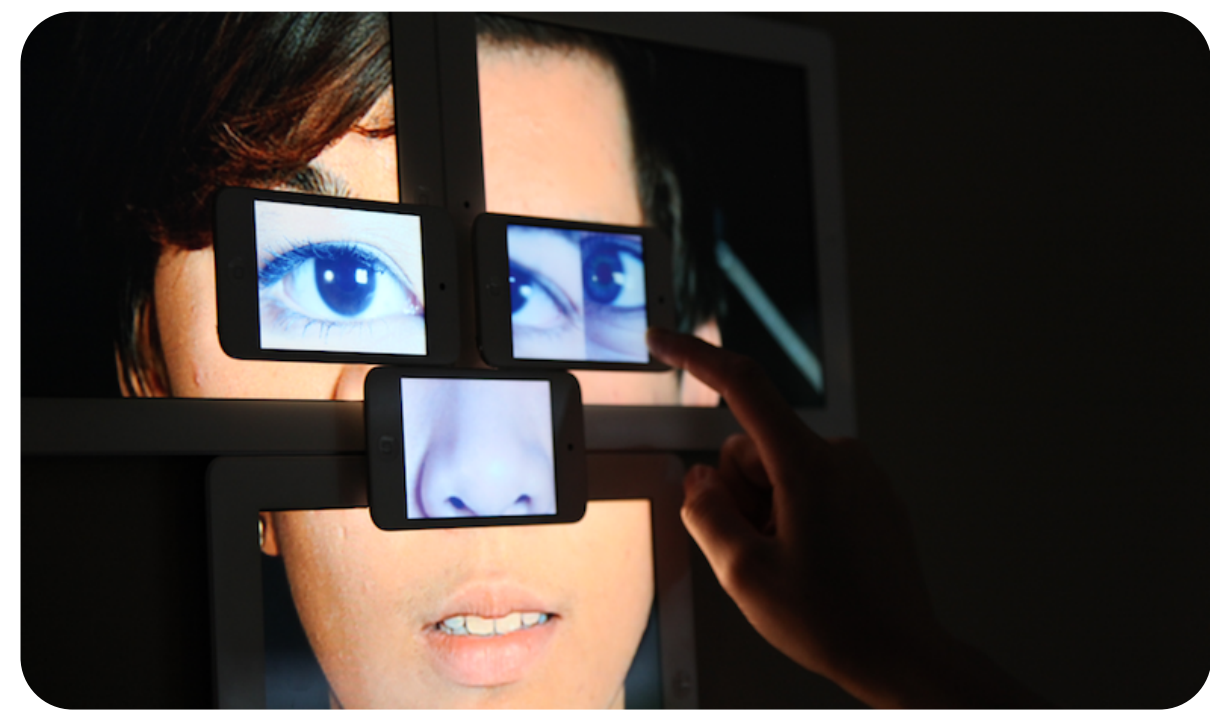

We present C4, an API designed specifically for multitouch mobile devices, which have limited computing power. C4 allows the programmer to work with media as first-class objects; it also provides techniques for easily integrating touch and gestural interaction, as well as rich animations, into user interfaces. At a technical level, the API is designed for efficient use and to have a smaller resource footprint for mobile devices, culminating in a prototyping language for the rapid development of creative mobile

\section{In This Issue:}

- C4: A Creative-Coding API for Media, Interaction and Animation

- MACS On Top: A Collaborative TabletopDiagraming Tool 


\section{MACS On Top: A Collaborative Tabletop-Diagraming Tool}

\section{By Christophe Bortolaso, Nicholas Graham, and Emmanuel Dubois}

Recent years have seen a profusion of new interactive systems combining physical and digital entities. These mixed systems put tangible artefacts into the user's hands, transforming the interaction into a real experience. Such interfaces are particularly relevant in public spaces such as museums, where they provide visitors with a fun and entertaining means of accessing knowledge. There are limitless ways in which physical and digital entities can be combined. However, there are few real examples of these systems, so each new design involves significant exploration and experimentation.

To assist in the design of mixed interactive systems, researchers from the University of Toulouse have developed a method called Model Assisted Creativity Sessions (MACS). This method supports brainstorming where participants from a wide range of backgrounds explore trade-offs in the

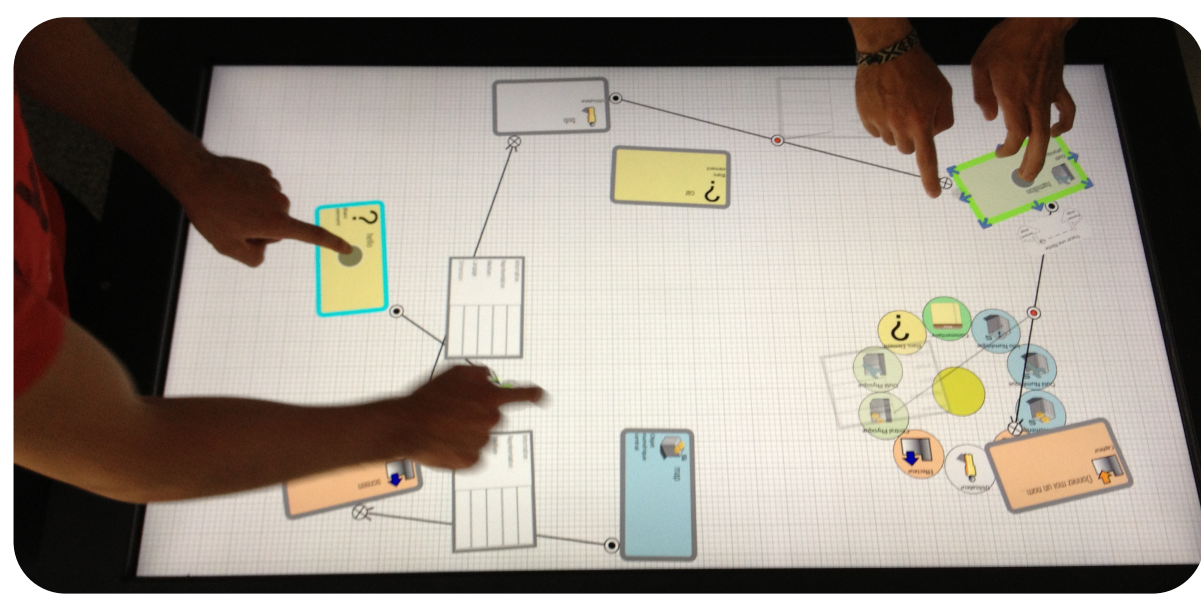

design of a mixed interactive system. To do so, they collaboratively use pens, sticky notes and large sheets of paper to draw diagrams representing a variety of candidate solutions.

We observed during design sessions that editing diagrams slows down the creative process. During sessions, participants often wish to explore several similar design alternatives. This requires them to recopy most of the diagram to produce a new version changed in small ways. However, most participants never do this, instead overlaying their new ideas on the same sheet of paper, rapidly making the diagram illegible.

To solve this problem, we built MACS On Top. This surface-based tool allows small groups of designers to collaboratively draw and edit diagrams, rapidly supporting creation and comparison of many design alternatives. To see MACS On Top in action, we have a demo video at: https://vimeo.com/52250192

\section{In the News:}

Thank you to everyone who contributed to

For SurfNet contact information please go to: www.nsercsurfnet.ca/pmwiki.php?n=SurfNet.Contact making our 2012 SurfNet Workshop in Waterloo such a success! In particular, thank you to our keynote speaker, Dr. Karon MacLean of UBC, who gave an extremely insightful and stimulating talk during our industry open house.

We had over 25 demonstrations running during our open house, showcasing work from across Canada, and all who attended considered it to be a big success. We've already had many local industry representatives following up with SurfNet researchers to explore opportunities for further work together.

Stay tuned for information on our 2013 SurfNet Workshop, which is tentatively scheduled for June in Alberta. In addition to our already successful workshop activities we will be bringing together current and potential partner organizations to discuss increased industrial interactions within SurfNet and explore exciting directions for "SurfNet 2.0". If you have some interesting ideas for where ICT and interactive technologies can support the future of your business, let us know and put aside some time in June next year to come share it with us!

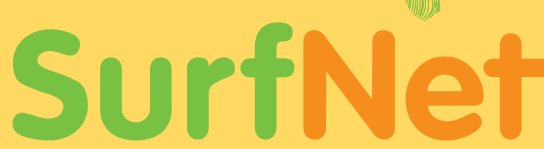

